\title{
Selective attention in peacocks during assessment of rival males
}

\author{
Jessica L. Yorzinski ${ }^{1,2, *}$, Gail L. Patricelli ${ }^{3}$, Siarhei Bykau ${ }^{4}$ and Michael L. Platt ${ }^{2,5,6,7}$
}

\begin{abstract}
Males in many species compete intensely for access to females. In order to minimize costly interactions, they can assess their rivals' competitive abilities by evaluating traits and behaviors. We know little about how males selectively direct their attention to make these assessments. Using Indian peafowl (Pavo cristatus) as a model system, we examined how males visually assess their competitors by continuously tracking the gaze of freely moving peacocks during the mating season. When assessing rivals, peacocks selectively gazed toward the lower display regions of their rivals, including the lower eyespot and fishtail feathers, dense feathers, body and wings. Their attention was modified based on the rivals' behavior such that they spent more time looking at rivals when rivals were shaking their wings and moving. The results indicate that peacocks selectively allocate their attention during rival assessment. The gaze patterns of males assessing rivals were largely similar to those of females evaluating mates, suggesting that some male traits serve a dual function in both intra- and intersexual selection. However, males spent more time than females looking at the upper eyespots and this could indicate that the upper eyespots function more in close-up rival assessment than mate choice.
\end{abstract}

KEY WORDS: Attention, Communication, Pavo cristatus, Sexual selection, Vision

\section{INTRODUCTION}

Sexual selection operates through both female mate choice (intersexual selection) and male-male competition (intrasexual selection; Darwin, 1871; Andersson, 1994). Both rival males and female mates may be interested in gaining similar information about a male. Therefore, in many species, the same male trait is used by both sexes (Borgia, 1979; Loffredo and Borgia, 1986; Zahavi, 1991; Berglund et al., 1996). For example, horns in mountain sheep (Geist, 1971), spurs in pheasants (von Schantz et al., 1989) and wing patches in dragonflies (Moore, 1990) are used in both male-male aggressive interactions and female choice. In species where a male trait is only evaluated by one of the sexes, it is often the males that are assessing the trait, suggesting that male traits often evolve initially in the context of male-male competition, and subsequently, in female choice (Berglund et al., 1996; Borgia and Coleman, 2000).

\footnotetext{
${ }^{1}$ Department of Wildlife and Fisheries Sciences, Texas A\&M University, College Station, TX 77843, USA. ${ }^{2}$ Duke Institute for Brain Sciences and Center for Cognitive Neuroscience, Duke University, Durham, NC 27710, USA. ${ }^{3}$ Department of Evolution and Ecology, University of California, Davis, CA 95616, USA. ${ }^{4}$ Bloomberg L.P., 731 Lexington Ave, New York, NY 10022, USA. ${ }^{5}$ Department of Neuroscience, Perelman School of Medicine, University of Pennsylvania, Philadelphia, PA 19104, USA. ${ }^{6}$ Department of Psychology, University of Pennsylvania, Philadelphia, PA 19104 USA. ${ }^{7}$ Marketing Department, Wharton School, University of Pennsylvania, Philadelphia, PA 19104, USA.

*Author for correspondence (jyorzinski@tamu.edu)

(D) J.L.Y., 0000-0002-4193-6695
}

Received 4 October 2016; Accepted 5 January 2017
In order to make these assessments, males and females must selectively direct their attention toward male traits. Because of constraints in sensory processing, animals cannot simultaneously attend to all aspects of their environment (Dukas, 2002); indeed, behavioral performance on a peripheral task declines when animals are focused on a central task, especially when the central task is difficult (Vreven and Blough, 1998; Dukas and Kamil, 2000). Because of this limited attention, animals need to direct their attention to extract the exact information they require (O'Brian and Showalter, 1993; Yorzinski et al., 2013; Yorzinski and Platt, 2014). We know little about how animals selectively direct their attention during mate and rival assessment (Dukas, 2002; Rosenthal, 2007). Previous work has shown that female peafowl shift their gaze between potential mates and their environment, potentially scanning for predators and other conspecifics while assessing mates. And, when evaluating a mate, peahens selectively direct their attention toward specific display regions of peacocks (Yorzinski et al., 2013). In contrast, we do not know how males selectively alter their attention when assessing other males.

We therefore investigated how males direct their attention when they assess potential rivals, using peacocks as a model system. Indian peafowl (Pavo cristatus) are a lekking species in which males establish display territories and females visit the males to select their mates (Petrie et al., 1991). Competition among peacocks is intense as mating success is highly skewed toward a small proportion of successful males. Males compete with each other by displaying their erect trains or walking parallel to other males. If aggression escalates, they chase each other and engage in fights that consist of them jumping and using their spurs (Petrie et al., 1991; Loyau et al., 2005). Males with longer trains and tarsi establish territories in central locations within leks and engage in more agonistic behaviors with other males. In contrast, males with shorter trains are less likely to establish display territories (Loyau et al., 2005). We monitored the gaze of freely moving peacocks as they evaluated the displays of other males to determine how they allocate their attention during rival assessment and whether their attention was influenced by the behavior of their rivals. We also compared their gaze patterns during rival assessment with the gaze patterns of females during mate evaluation.

\section{MATERIALS AND METHODS}

We analyzed the gaze behavior of 14 adult peacocks (Pavo cristatus Linnaeus 1753) during the mating season (April and May of 2012 and 2013) in Durham, NC, USA $\left(36.01^{\circ} \mathrm{N}, 79.02^{\circ} \mathrm{W}\right)$. The males were housed in an enclosure (92 $\mathrm{m}$ perimeter) that was $\sim 400 \mathrm{~m}$ from the enclosure ( $46 \mathrm{~m}$ perimeter) in which the females were regularly housed; the males and females therefore did not physically interact with each other (with the exception of experiments conducted in Yorzinski et al., 2013). For each trial, a focal male was outfitted with a telemetric eye-tracker (Yorzinski et al., 2013; Yorzinski et al., 2015). The eye-tracker consisted of a transmitter (Iscan, Woburn, MA, USA; $345 \mathrm{~g}$ ) connected to a headpiece (designed by Positive Science, LLC, New York, NY, USA; 25 g) that had two cameras that recorded the bird's eye and the scene in front of the bird (see Yorzinski et al., 2013 for further details on the eye-tracker). 
The transmitter sent both video images ( 30 frames $\mathrm{s}^{-1}$ ) to a nearby receiver. The receiver was connected to a DVD recorder (Toshiba D-R410) that recorded the video signals and then passed the signals to a computer (Dell Dimension 2300). The computer processed the video signals with custom eye-tracking software (Iscan) and displayed the videos. The eye-tracker does not prevent birds from looking at elevated targets or holding their heads upright; birds exhibit natural mating behavior while wearing the eye-tracker (Yorzinski et al., 2013). Because the eye-tracker recorded the eye movements of only one of the bird's eyes, a patch was placed on the bird's other eye to ensure that we were monitoring all the visual information that the bird was using to assess their rivals. Birds were trained wearing the patch along with the headgear and exhibited normal behaviors even when one eye was covered. The males were habituated to wearing the eye-tracker in daily sessions of about $60 \mathrm{~min}$ for several weeks prior to the trial. After the eye-tracker was calibrated using an oculometric approach based on corneal reflections ( $<5$ deg error; Yorzinski et al., 2013), the focal male was put inside a testing enclosure $(27 \mathrm{~m}$ perimeter $)$ that contained another peacock ('rival') and two peahens (there were therefore 4 birds in the testing enclosure during each trial). The peahens were transported from the female enclosure to a holding enclosure $(20 \mathrm{~m}$ perimeter) beside the testing enclosure at least 1 day before the trials began so that the females could habituate to the new area (they were not able to see males when they were in the holding enclosure); they were then relocated to the testing enclosure immediately before a trial. The focal peacock was allowed to move freely within the testing enclosure for $1-1.5 \mathrm{~h}$. The behavior of the birds was continuously recorded using external camcorders (Sony SR47) placed in the corners of the testing enclosure. Each of the 14 focal peacocks was tested in one to four different trials (mean: $2.0 \pm 0.21$ trials) for a total of 28 trials (20 trials recorded the left eye and 8 trials recorded the right eye of the birds). The experiments in this study were approved by Duke University (IACUC: A169-11-07).

\section{Gaze and behavioral extraction}

We isolated clips $(N=230)$ from the trial recordings in which the focal male was directing his gaze toward the displaying male rival. Because the focal males did not often display (likely because they were wearing the eye-tracker), we only selected clips in which the focal male was not displaying. A clip began when the focal male directed his gaze toward a displaying rival male and ended when he looked away from the rival male for at least $10 \mathrm{~s}$. A single clip could therefore include sequences when the focal male directed his gaze toward the rival male, then looked at the environment, females, or himself for less than $10 \mathrm{~s}$, and then returned to looking at the rival male (mean \pm s.e.m. clip length: $40.5 \pm 2.7 \mathrm{~s})$. The videos (30 frames $\mathrm{s}^{-1}$ ) were deinterlaced to generate 60 fields $\mathrm{s}^{-1}$ (Turbo.264, Elgato Systems, San Francisco, CA, USA). The gaze data were adjusted for parallax errors (Maurer, 1975; Yorzinski and Platt, 2014), which exist because the scene camera cannot be perfectly aligned with the eye of the bird without physically blocking the bird's vision. Out of these clips, the rival male was directly displaying toward a female ('directed') in $65 \%$ of the clips; in the remaining $35 \%$ of clips, the rival male was displaying but the display was not directed toward a female ('non-directed'). In the directed clips, a female was within $\sim 3 \mathrm{~m}$ of the rival male and the rival male was tracking the female by shifting his display relative to her movement by keeping his train perpendicular to her body; in the non-directed clips, a female was not within $\sim 3 \mathrm{~m}$ of the rival male and the rival male was not adjusting his display relative to the movement of the female.
All of the clips were analyzed field-by-field using custom MATLAB scripts to assign each gaze coordinate to a target. The targets included whether the gaze was directed toward the rival male, one of the females, the focal male, or the environment. If the target was the rival male or the females, the gaze coordinate was assigned to a region-of-interest (ROI; see Results for ROI demarcations). We used the same ROI designations from our previous work (Yorzinski et al., 2013) such that there were 9 ROIs in the rivals' frontal display (body, head/crest, scale feathers, legs, dense feathers, lower eyespots, lower fishtails, upper eyespots, upper fishtails) and 10 ROIs in the rivals' backside display (black feathers, white feathers, wings, tail, legs, dense feathers, lower eyespots, lower fishtails, upper eyespots, and upper fishtails). We designated 6 ROIs in the female (head/crest, neck, chest, wings/ body, tail, and legs).

In addition, the behavior of the males and females during the clips was analyzed. The videos from the external camcorders were imported into video editing software (Final Cut Express, Apple, Cupertino, CA, USA) and synchronized with the eye-tracking videos. We synchronized them by aligning frames in which birds made distinct movements that were visible in both videos. The behaviors analyzed were wing shaking (vertical movement of the wings), movement (walking or stepping) and train rattling (shaking the iridescent feathers and producing a rattle-like sound) of the rival male, movement of the focal male and females (walking, running or stepping), as well as the distance between the focal male and rival male. We estimated the distance between the focal and rival male by measuring the width of the rival male's body (in pixels) in the eye-tracker scene camera; we determined the width of a similarly sized object at known distances and then used this conversion to estimate the distance between the males. Because the focal male and rival male were within $4 \mathrm{~m}$ of each other in $93 \%$ of the clips, the distance was categorized as $<2 \mathrm{~m}$ and $2-4 \mathrm{~m}$. For each of the behaviors, we assessed whether or not the behavior was occurring in each field of the video clip. Train rattling only occurred during clips when the rival male was performing a directed display. Train shivering (Dakin et al., 2016) only occurred in four of the nondirected display clips and we did not therefore analyze this behavior. Because focal males only looked at females in $9 \%$ of the non-directed display clips, we only analyzed female movement during directed clips, in which males looked at females in $62 \%$ of the clips.

\section{Morphological features}

In order to investigate the relationship among morphological features of peacocks, we obtained photographs $(N=30)$ of peacocks directly facing the camera and displaying from online sources and our study birds. We measured the width and height of the train in pixels (Adobe Photoshop 7.0, San Jose, CA, USA) and then converted these values to $\mathrm{mm}$ (the average width of the peacock's blue body is $155 \mathrm{~mm}$, which was used as a conversion factor). We also counted the number of lower eyespot feathers (eyespot feathers below a horizontal line directly above the scale feathers) and upper eyespot feathers (eyespot feathers above a horizontal line directly above the scale feathers; see Results for further details on these morphological measurements).

\section{Statistical analysis}

We performed repeated-measures mixed linear models (PROC mixed) to compare whether the percentage of time that focal males directed their attention toward different targets varied based on whether the rival male was performing a directed or non-directed 
display. We analyzed overall gaze patterns with respect to ROIs using a generalized linear mixed model (GLMM) for the rival males' frontal display and backside display as well as the female analyses (the model was similar to that used in eqn 1 in Yorzinski et al., 2013). For each analysis, we modeled the number of counts in each ROI as drawn from an over-dispersed Poisson distribution and used a log link function. The ROIs were categorical regressors, focal male and rival male identity were zero-mean random effects, and the logarithm of total clip duration and the logarithm of total ROI size (as a fraction of total average male size) were constant offsets. By including the size of the ROIs within the model, we accounted for variation in the surface area of different ROIs. We excluded one ROI (black feathers) from the backside display model because it had zero gaze counts in all recorded trials. We also re-ran this analysis using the rival male as the sampling unit rather than focal male. To investigate whether the eye that was being monitored by the eyetracker influenced the results, we re-ran these models separately for the left and right eye. In addition, we re-ran the models separately for whether the rival male was performing a directed or non-directed display; because of insufficient data, it was not possible to perform the analyses on non-directed display clips for the backside and female analyses. Model fits were performed using Markov Chain Monte Carlo methods in JAGS via the 'rjags' package in R. Data, model files and additional plots are available at https://github.com/ bykau/peacock.

We performed mixed linear models (PROC mixed) to compare whether males (this study) and females (previous study; Yorzinski et al., 2013) differed in the percentage of time they spent viewing ROIs in the frontal and backside displays; the dependent variable was the percentage of time that males or females spent gazing at each ROI; the independent variables were the sex of the focal bird, ROI and their interaction (the ID of the focal bird was a random variable). It is possible that males spent more time looking at the upper eyespots compared with females (see Results) because males are taller than females ( $\sim 100 \mathrm{~mm}$ taller) and their area centralis may project slightly higher than that of females. In order to test for this possibility, we re-ran this analysis a second time but modified the upper eyespot ROI such that it excluded the lower part of the upper eyespots (the horizontal line that forms the lower boundary of the upper eyespot ROI was shifted upwards by $100 \mathrm{~mm}$ ).

We performed mixed linear models (PROC mixed) to assess whether behaviors impact the focal males' gaze. The dependent variable was the percentage of time that the focal males spent gazing at the rival males (compared with gazing at the environment, themselves or females). The independent variables were whether the behavior was occurring or not, the identity of the focal male and rival male, the clip type (directed or non-directed) and the interaction between behavior and clip type. The behaviors were wing shaking, movement and train rattling of the rival male, movement of the focal male and females, as well as the distance between the focal male and rival male. Linear regressions were performed to assess morphological relationships (train width versus train height and lower eyespots versus upper eyespots). The linear mixed models and regressions were performed using SAS (Cary, $\mathrm{NC}$, USA); means \pm s.e.m. are reported.

\section{RESULTS}

During our sample clips, focal males spent $27.9 \%$ of their time gazing at the displaying rivals. They spent $43.4 \%$ of their time gazing at the environment, as well as $3.1 \%$ and $0.5 \%$ of their time looking at females and themselves, respectively. Because of errors with the eye-tracking system (e.g. transmission problems and battery failure), $25.1 \%$ of gaze data could not be analyzed. Based on the data that could be analyzed, focal males allocated their attention toward targets differently depending on whether the rivals' display was directed or non-directed (Fig. 1A,B). Focal males spent more time gazing at the rival males' frontal display $\left(F_{1,13}=29.77\right.$, $P=0.0001)$ and less time looking at the females $\left(F_{1,13}=31.12\right.$, $P<0.0001)$ and environment $\left(F_{1,13}=19.18, P=0.0007\right)$ during nondirected displays compared with directed displays. The amount of time they spent looking at the rival males' backside display $\left(F_{1,13}=0.54, P=0.47\right)$ and themselves $\left(F_{1,13}=2.28, P=0.15\right)$ was not affected by the type of display (Fig. 1C).

When focal males directed their attention toward their rivals, their gaze was directed toward specific display areas (Table S1; Fig. 2). During the rivals' frontal display, the focal males spent more time than random expectation (based on the surface area of the ROIs) looking at the dense feathers, lower eyespots, lower fishtails, legs and body, and less time looking at the upper eyespots and upper fishtails; the amount of time they gazed toward the head, crest, and scales was not different than expected (Table 1, Frontal; Fig. 3A). During the rivals' backside display, the focal males spent more time than random expectation gazing at the lower eyespots, lower fishtails and wings, and less time gazing at the black feathers and tail; the amount of time they gazed at the other backside display regions (upper eyespots, upper fishtails, white feathers, dense feathers and legs) was not different than expected (Table 1,


Fig. 1. Peacocks allocate their attention toward targets differently depending on whether a rival's display is directed or non-directed. $(A, B)$ Fraction of time allocated to various targets during $(A)$ directed or $(B)$ nondirected rival displays. (C) Bar graph of percentage of time allocated to targets during directed and non-directed rival displays. Peacocks spend more time looking at rival males during frontal displays and less time looking at females and the environment when the rival displays are directed compared with nondirected. Values are means+s.e.m; ${ }^{*} P<0.05$ 
A

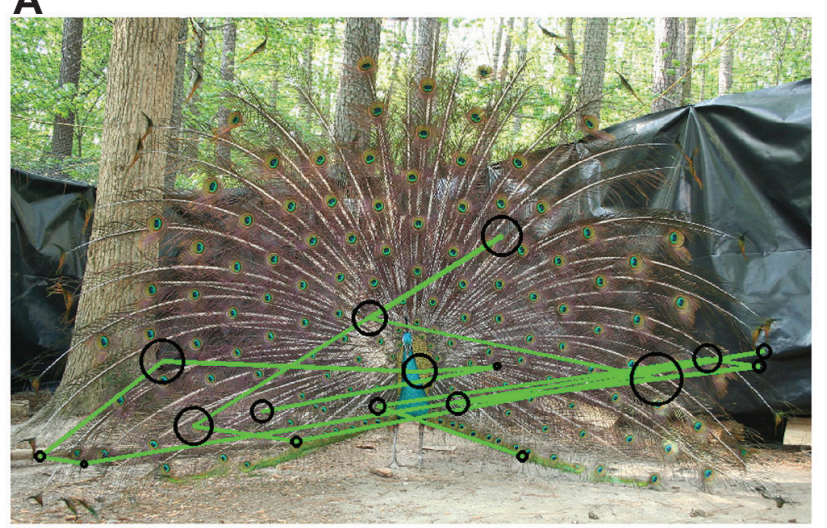

C

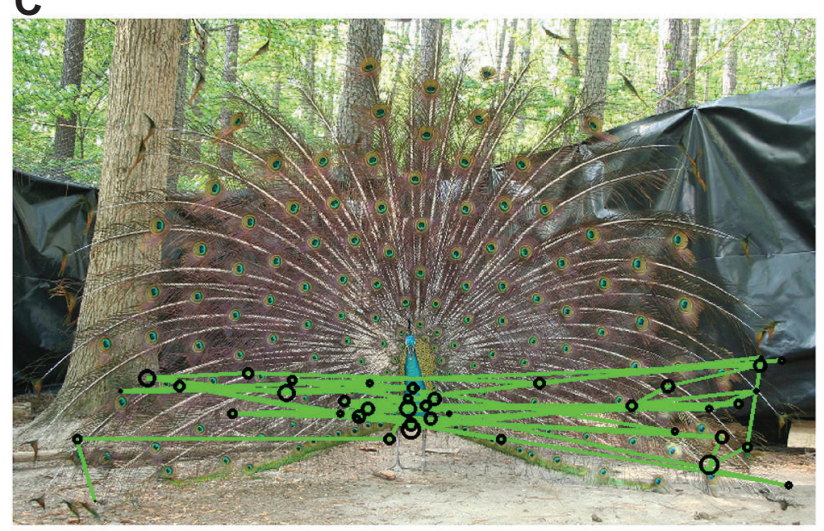

$\mathbf{F}$

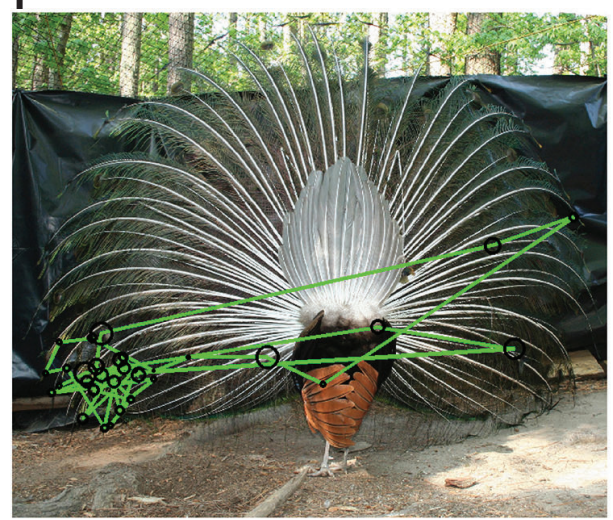

B



D

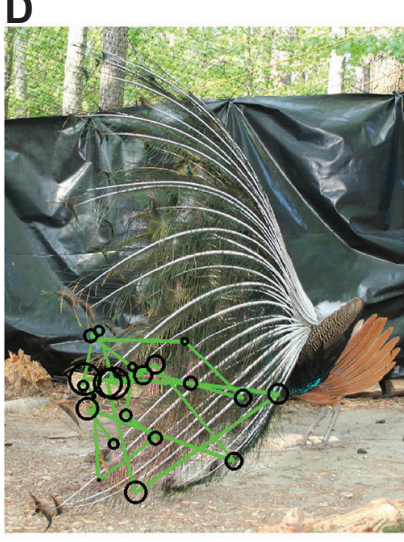

E
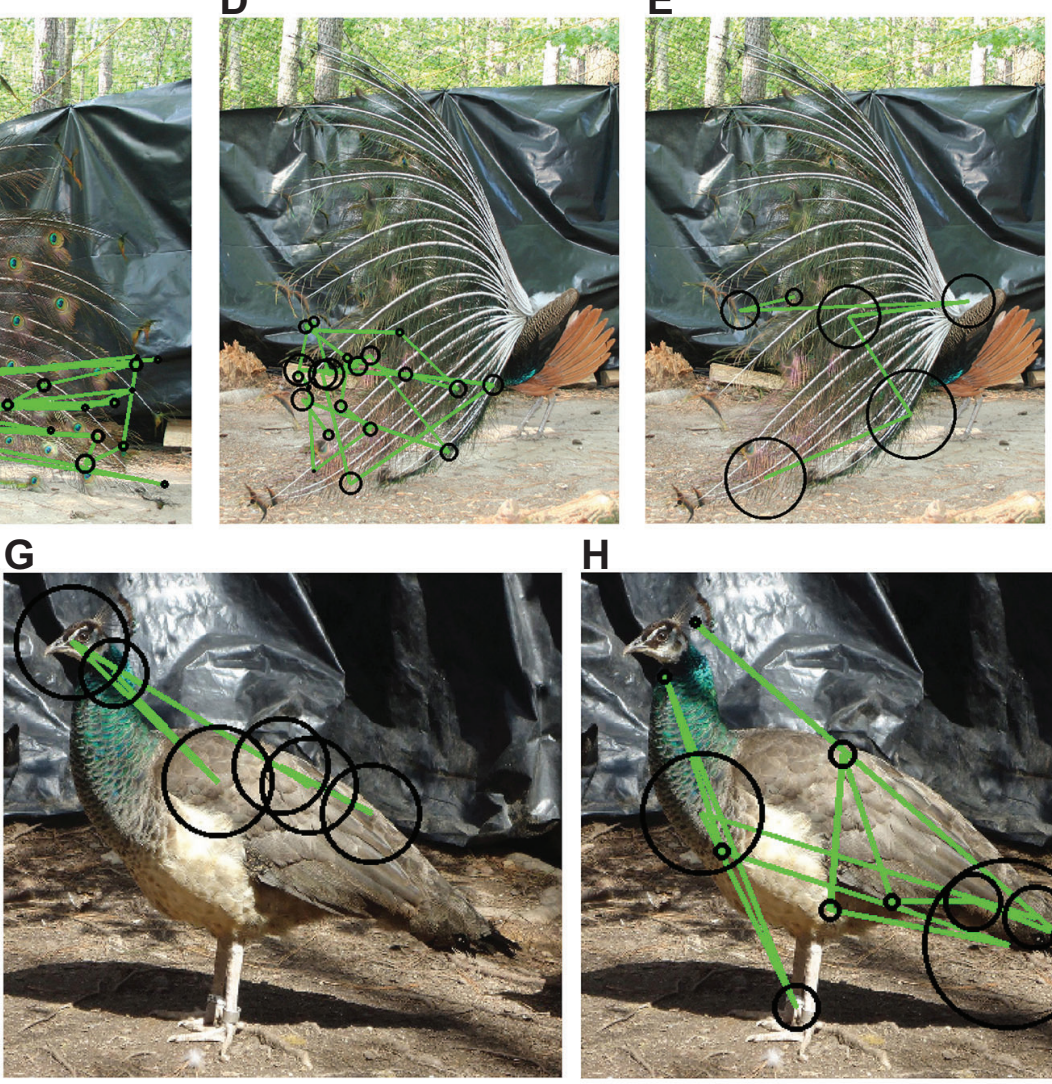

H

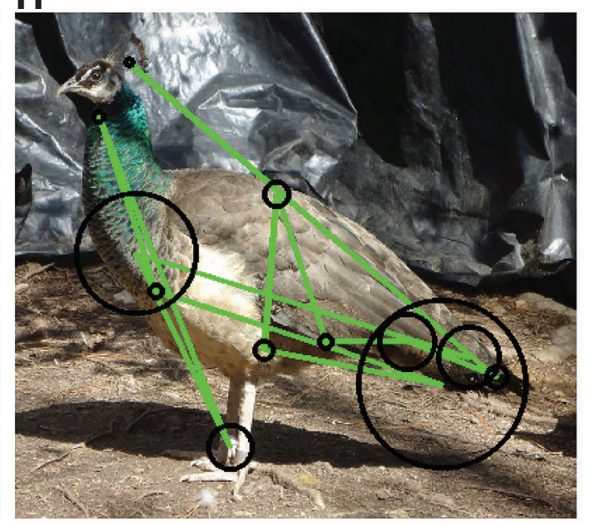

Fig. 2. Peacocks direct their attention toward specific rival display areas and specific areas of peahens. Representative scan paths of different focal males showing visual assessment of their rival's frontal $(A-C)$ and backside $(D-F)$ display as well as a female $(G, H)$. B and $F$ are scanpaths from the same male; the other scanpaths are each from different males. The size of the black circles indicates the relative amount of time males spent looking at each location.

Backside; Fig. 3B,C). These frontal and backside display results were similar regardless of whether the rivals' displays were directed or non-directed (Table S2) and when the analysis was re-run using the rival male as the sample unit (Table S3). Compared with female gaze patterns (data from Yorzinski et al., 2013), males spent a larger percentage of time looking at the upper eyespots and less time looking at the dense feathers in the frontal display and less time looking at the dense feathers in the backside display (Table 2). Even when accounting for males being taller than females, males still tended to spend more time looking at the upper eyespots compared with females $(t=2.40$, d.f. $=342, P=0.017)$. When males directed their gaze toward females, the amount of time they spent looking at different regions on the female did not differ from random expectation (Table 1, Female; Fig. 3D).
Based on measurements taken from photographs of displaying peacocks facing directly toward the camera $(N=30)$, the width of peacocks' trains was positively correlated with the height of peacocks' trains $\left(F_{1,28}=44.55, R^{2}=61 \%, P<0.0001\right.$; Fig. 4A). Furthermore, the number of lower eyespots was negatively correlated with the number of upper eyespots $\left(F_{1,28}=13.39, R^{2}=32.4 \%, P=0.001\right.$; Fig. 4B). Variation among peacocks in the total number of lower and upper eyespots was relatively small (mean: $154 \pm 1.5$ feathers; range: 130-169 feathers; first quartile: 148 feathers; third quartile: 160 feathers) and similar to the total reported in previous studies (reviewed in Dakin and Montgomerie, 2011).

We found that males' gaze behavior was impacted by the behavior of their potential rivals (Table S4; Fig. 5). Focal males spent more time gazing at their rivals (compared with the 
Table 1. Model coefficients and confidence intervals for focal male gaze with respect to rival male (frontal and backside display) and female regions of interest (ROIs)

\begin{tabular}{|c|c|c|c|c|c|c|c|c|c|}
\hline \multirow[b]{2}{*}{ ROI } & \multicolumn{3}{|c|}{ Both eyes } & \multicolumn{3}{|c|}{ Left eye } & \multicolumn{3}{|c|}{ Right eye } \\
\hline & $B$ (median) & $2.5 \%$ & $97.5 \%$ & $B$ (median) & $2.5 \%$ & $97.5 \%$ & $B$ (median) & $2.5 \%$ & $97.5 \%$ \\
\hline \multicolumn{10}{|l|}{ Frontal } \\
\hline Body & 1.51 & 0.65 & 2.41 & 1.46 & 0.37 & 2.38 & 2.62 & 0.88 & 4.41 \\
\hline Head/crest & 0.10 & -0.90 & 1.11 & 0.80 & -0.36 & 1.84 & -2.00 & -4.61 & 0.18 \\
\hline Scale feathers & -0.14 & -1.10 & 0.86 & 0.41 & -0.73 & 1.52 & -1.90 & -4.24 & -0.09 \\
\hline Legs & 1.20 & 0.29 & 1.97 & 0.82 & -0.16 & 1.96 & 1.96 & 0.22 & 3.55 \\
\hline Dense feathers & 1.21 & 0.36 & 2.07 & 1.10 & -0.03 & 2.18 & 1.53 & -0.19 & 3.25 \\
\hline Lower eyespots & 1.20 & 0.41 & 2.11 & 1.16 & 0.21 & 2.18 & 1.75 & 0.11 & 3.47 \\
\hline Lower fishtails & 1.04 & 0.15 & 1.92 & 0.96 & 0.05 & 1.89 & 1.59 & -0.02 & 3.28 \\
\hline Upper eyespots & -2.37 & -3.25 & -1.61 & -2.77 & -3.78 & -1.76 & -1.69 & -3.38 & 0.12 \\
\hline Upper fishtails & -3.75 & -4.64 & -2.89 & -3.91 & -4.97 & -2.84 & -3.80 & -5.54 & -2.06 \\
\hline \multicolumn{10}{|l|}{ Backside } \\
\hline Black feathers & - & - & - & - & - & - & - & - & - \\
\hline White feathers & -0.78 & -2.59 & 0.92 & -1.87 & -4.15 & -0.02 & 1.37 & -1.99 & 4.38 \\
\hline Wings & 3.33 & 2.08 & 4.81 & 3.28 & 1.83 & 4.85 & 2.28 & -0.76 & 5.35 \\
\hline Tail & -3.65 & -5.81 & -1.67 & -3.96 & -6.28 & -1.98 & -2.30 & -5.93 & 1.29 \\
\hline Legs & -1.47 & -3.67 & 0.29 & -1.87 & -3.99 & 0.12 & -0.94 & -5.47 & 2.71 \\
\hline Dense feathers & 0.09 & -1.60 & 1.69 & 0.94 & -0.66 & 2.50 & -0.72 & -4.88 & 2.62 \\
\hline Lower eyespots & 2.55 & 1.15 & 3.94 & 2.87 & 1.49 & 4.32 & 2.12 & -0.61 & 4.96 \\
\hline Lower fishtails & 2.61 & 1.11 & 4.13 & 2.97 & 1.48 & 4.55 & 2.81 & 0.01 & 5.60 \\
\hline Upper eyespots & -1.37 & -2.91 & 0.09 & -1.36 & -2.93 & 0.36 & -2.41 & -5.88 & 1.01 \\
\hline Upper fishtails & -1.29 & -2.85 & 0.25 & -0.92 & -2.62 & 0.63 & -1.77 & -5.54 & 1.25 \\
\hline \multicolumn{10}{|l|}{ Female } \\
\hline Head/crest & -0.73 & -1.63 & 0.16 & -0.88 & -2.17 & 0.29 & -0.58 & -2.34 & 0.93 \\
\hline Neck & -0.16 & -0.97 & 0.65 & -0.21 & -1.36 & 0.96 & -0.61 & -2.09 & 0.95 \\
\hline Chest & 0.27 & -0.56 & 1.06 & 0.38 & -0.67 & 1.56 & 0.24 & -1.19 & 1.79 \\
\hline Wings/body & 0.40 & -0.40 & 1.20 & 0.28 & -0.87 & 1.42 & 0.93 & -0.43 & 2.42 \\
\hline Tail & 0.36 & -0.45 & 1.24 & 0.57 & -0.57 & 1.71 & 0.02 & -1.49 & 1.46 \\
\hline Legs & -0.10 & -0.87 & 0.69 & -0.12 & -1.23 & 0.98 & 0.01 & -1.52 & 1.51 \\
\hline
\end{tabular}

environment, themselves or females) when their rivals were shaking their wings and moving. The focal males also spent more time looking at their rivals when the focal males were stationary rather than moving and when the females were moving. In contrast, the
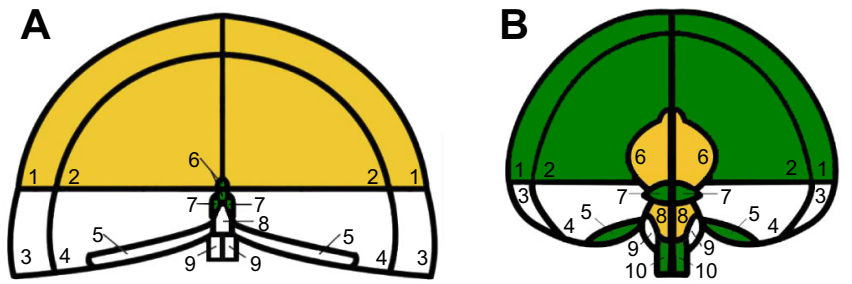

C

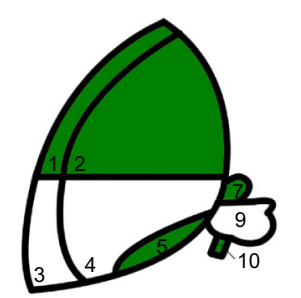

D

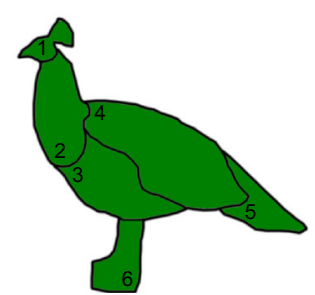

Fig. 3. Distribution of peacock gaze toward displaying rivals and peahens. The probability that focal peacocks gazed at specific regions of interest (ROIs) in the rival male's frontal $(A)$ and backside $(B, C)$ display as well as the female (D). White, yellow and green shading indicates areas viewed at levels greater than expected by chance, less than expected by chance and not different from chance, respectively. 1, upper fishtails; 2 , upper eyespots; 3 , lower fishtails; 4, lower eyespots; 5 , dense feathers. In A: 6, head and crest; 7 , scale feathers; 8 , body; 9 , legs. In B and C: 6 , tail; 7 , white feathers; 8 , black feathers; 9 , wings; 10 , legs. In D: 1 , head and crest; 2 , neck; 3 , chest; 4 , wings and upper body; 5 , tail; 6 , legs. amount of time that focal males spent looking at their rivals did not vary depending on the distance between the males or whether the rival male was train rattling. These results were qualitatively similar when analyzed with non-parametric methods (Wilcoxon signedrank test) except that focal males did not spend significantly more time looking at rival males when the focal males were stationary. The amount of time that focal males gazed at rivals varied depending on the specific focal male and rival during the wing shaking, train rattling, female movement and distance analyses. In most cases, focal males gazed at male rivals more during nondirected clips versus directed clips.

\section{DISCUSSION}

Peacocks selectively shifted their gaze toward specific display regions of their displaying rivals. They focused on the lower display feathers (eyespots and fishtails), dense feathers, body and wings. They also directed more attention toward their rivals when the rivals were shaking their wings and moving but not when they were train rattling. During our observation periods, peacocks spent over a third of their time gazing at their displaying rivals, allowing them to monitor their rivals' traits and behaviors. Because our sample periods began when focal males gazed at displaying rivals (and continued until focal males looked elsewhere for $>10 \mathrm{~s}$ ), this percentage may be higher than if we had recorded focal male gaze during the total time rivals were displaying. However, it is clear from these sample periods that males spend a significant fraction of their time monitoring their rivals.

\section{Rival traits and behavior}

Peacocks focused their attention on specific traits of their rivals. They directed most of their gaze toward the lower eyespot feathers, 
Table 2. Comparison between male (this study) and female (previous study by Yorzinski et al., 2013) gaze patterns as they evaluate a displaying male rival or mate for the frontal and backside display

\begin{tabular}{|c|c|c|c|c|}
\hline ROI & Males & Females & $t$ & $P$-value \\
\hline \multicolumn{5}{|l|}{ Frontal } \\
\hline Body & $5.7(1.1)$ & $3.2(1.6)$ & 1.36 & 0.18 \\
\hline Head/crest & $1.0(1.1)$ & $0.0(1.6)$ & 0.52 & 0.60 \\
\hline Scale feathers & $1.9(1.1)$ & $0.6(1.6)$ & 0.69 & 0.49 \\
\hline Legs & $5.3(1.1)$ & $4.7(1.6)$ & 0.32 & 0.75 \\
\hline Dense feathers & $8.4(1.1)$ & $14.6(1.6)$ & 3.31 & $0.001^{*}$ \\
\hline Lower eyespots & $46.7(1.1)$ & $49.9(1.6)$ & 1.72 & 0.09 \\
\hline Lower fishtails & $19.1(1.1)$ & $22.3(1.6)$ & 1.69 & 0.09 \\
\hline Upper eyespots & $9.3(1.1)$ & $2.7(1.6)$ & 3.45 & $0.0006^{*}$ \\
\hline Upper fishtails & $2.7(1.1)$ & $2.0(1.6)$ & 0.38 & 0.70 \\
\hline \multicolumn{5}{|l|}{ Backside } \\
\hline Black feathers & $0.0(2.1)$ & $0.0(1.8)$ & 0.00 & 1.00 \\
\hline White feathers & $1.5(2.1)$ & $0.5(1.8)$ & 0.35 & 0.72 \\
\hline Wings & $14.6(2.1)$ & $11.9(1.8)$ & 0.99 & 0.32 \\
\hline Tail & $0.6(2.1)$ & $0.3(1.8)$ & 0.12 & 0.90 \\
\hline Legs & $2.3(2.1)$ & $2.6(1.8)$ & 0.13 & 0.89 \\
\hline Dense feathers & $2.6(2.1)$ & $16.5(1.8)$ & 4.95 & $<0.0001^{*}$ \\
\hline Lower eyespots & $39.8(2.1)$ & $41.0(1.8)$ & 0.41 & 0.68 \\
\hline Lower fishtails & $28.4(2.1)$ & $21.3(1.8)$ & 2.54 & 0.012 \\
\hline Upper eyespots & $6.3(2.1)$ & $4.8(1.8)$ & 0.53 & 0.59 \\
\hline Upper fishtails & $3.8(2.1)$ & $1.2(1.8)$ & 0.95 & 0.34 \\
\hline
\end{tabular}

Least squares means of the percentage of time (and standard error) are displayed for each ROI of males and females as well as the $t$ statistics and $P$-values comparing the sexes (d.f. $=304$ for frontal analysis and d.f. $=297$ for backside analysis). Asterisks indicate significant difference (using a Bonferroni correction) in the percentage of time that males versus females spend looking at a particular ROI of a displaying male.

lower fishtail feathers, dense feathers and legs. Interestingly, previous work found that train and tarsi length in peacocks were important traits during intrasexual selection. Males with longer trains and tarsi established territories in central locations within leks and engaged in more agonistic behaviors with other males (Loyau et al., 2005). Given that peacocks were gazing along the bottom portions of their rivals' displays and their rivals' legs, they could have been assessing the width of the trains (which is positively correlated with the length of the train) and length of the tarsi. In addition, we found a negative relationship between the number of eyespots in the lower and upper train. It would be interesting to determine whether peacocks that invest in more eyespots in the lower train versus the upper train are more successful in deterring rivals given that male gaze is directed toward the lower display regions and the total number of eyespot feathers is fairly similar across males (this study; Dakin and Montgomerie, 2011). Peacocks also often gazed toward the body of their rivals. By directing their attention toward the bodies as well as the legs, peacocks could have been monitoring their rivals for aggressive movements.

Peacocks' attention toward their rivals also varied depending on their rivals' behavior. Peacocks spent more time looking at their rivals when the rivals were moving and shaking their wings. Because movement could potentially lead to aggressive behavior (such as a chase), it is likely to be important for peacocks to monitor any movement so that they can respond quickly and appropriately. The wing shaking behavior could be an indicator of a rival's physical abilities if the speed and frequency of wing shaking indicates male quality. Physical performance, including strength, endurance and agility, can impact the outcome of contests among males in some species (McCullough and Simmons, 2016). If the wing shaking behavior reflects a rival's physical abilities, then attention toward this trait could provide males with an assessment of their rivals without

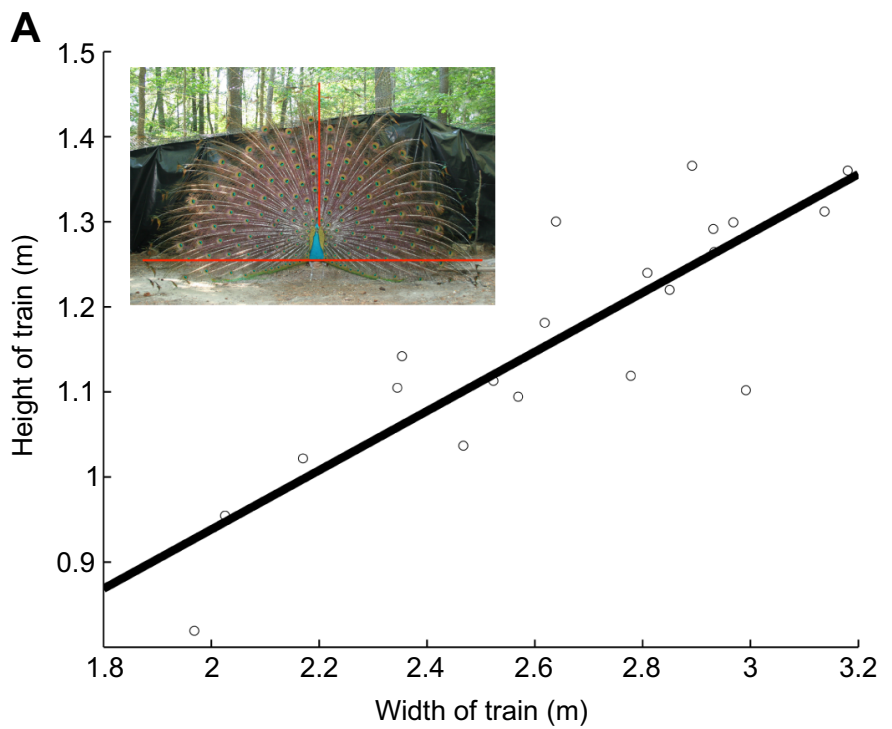

B

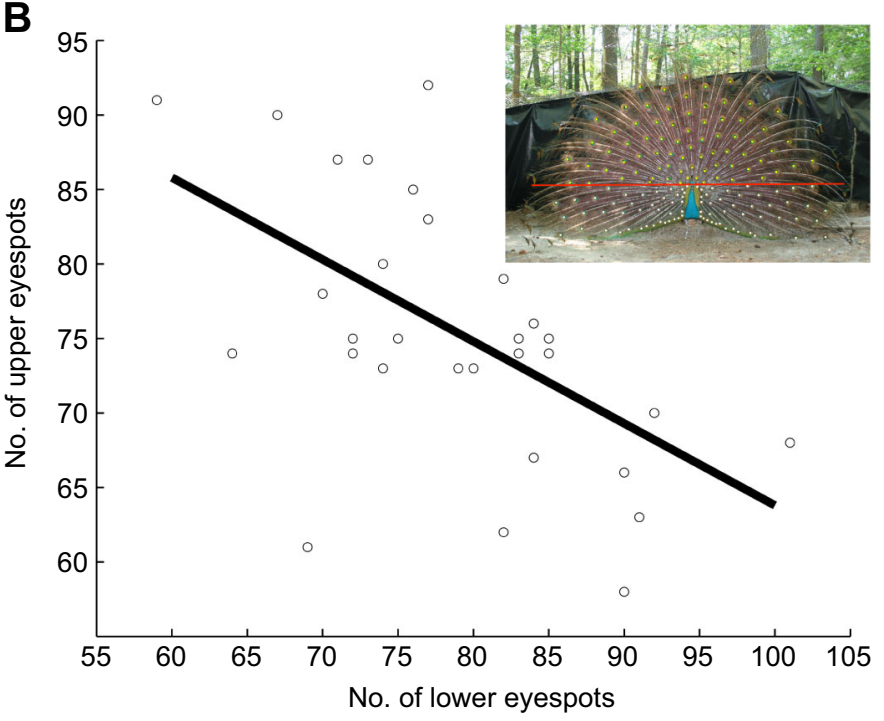

Fig. 4. Morphological features of peacock trains. (A) The width of peacock trains (horizontal red line in insert) is positively correlated with the height of peacock trains (vertical red line). (B) The number of lower eyespots (dots below horizontal red line in insert) is negatively correlated with the number of upper eyespots (dots above red line).

needing to engage in costlier contests. Further research is needed to investigate whether wing shaking is related to male condition or fighting ability. Interestingly, males did not direct more attention toward their rivals when their rivals were train rattling, suggesting that train rattling is not under intrasexual selection.

\section{Female and self assessment}

While assessing their competitors, peacocks did not spend very much time looking at females. In fact, they allocated less than $5 \%$ of their time during our sample periods gazing at females. It is possible that focal males did not often attend to females because these males were outfitted with the eye-tracking equipment. Males wearing the eye-tracker rarely displayed or attempted to copulate with females. Further research will be necessary to determine whether displaying males exhibit similar gaze patterns as non-displaying males. However, non-displaying males may also need to stay highly 


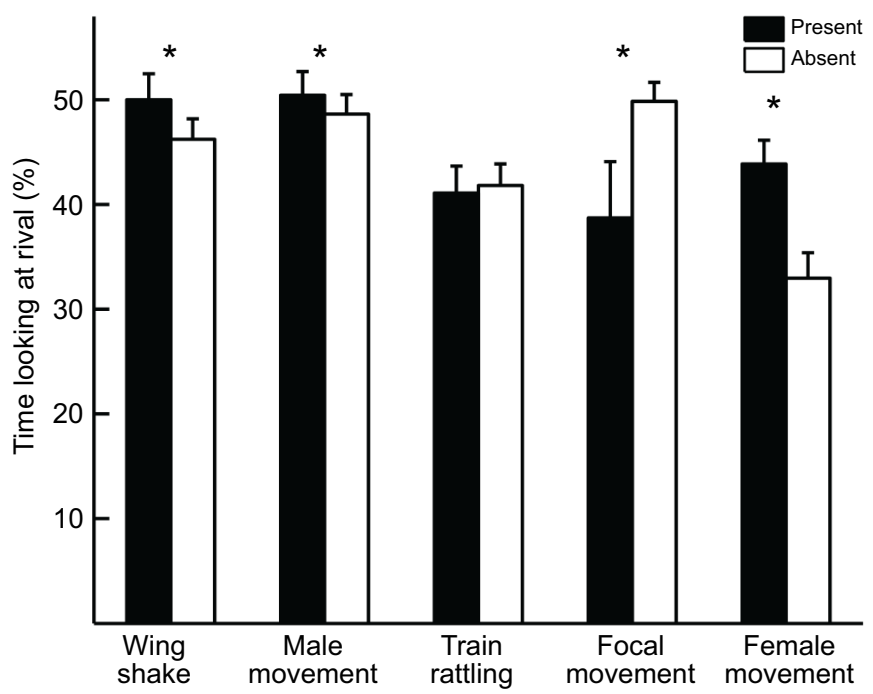

Fig. 5. Time peacocks spend looking at rivals depending on the behavior of rivals, females and themselves. Focal males spend more time looking at their rivals when the rivals are shaking their wings and moving but not when they are rattling their feathers. They also spend more time looking at their rivals when the focal males are stationary and the females are moving. Values are means+s.e.m; ${ }^{*} P<0.05$

focused on their rivals to avoid possible injury; they may only direct attention toward females when they are directly engaged in courtship displays. Further experiments will be necessary to determine how much time males allocate to monitoring females while they are courting them. We found that when males directed their gaze toward females, they evenly distributed their gaze among different female regions. While the females are mostly brown and white, they have iridescent feathers on their neck and crest. Because males were not preferentially gazing at these iridescent feathers, they may not use these traits when assessing female mating partners. These iridescent feathers may instead be targets of selection among females or a by-product of selection on iridescence in males.

Males allocated a small amount of time (less than 1\%) toward looking at their own train (as well as their feet in one clip). Even though the focal peacocks were not displaying, it is probably still important for them to maintain the appearance and condition of their feathers. Birds with ornamental plumage spend more time maintaining their plumage than birds without ornamental plumage (Walther and Clayton, 2005). And, female budgerigars prefer males that maintain their plumage by preening (Griggio et al., 2010). Peacocks also devote a significant amount of their daily time budget to preening (Walther, 2003) and directing attention toward themselves could allow them to monitor the condition of their feathers.

\section{Dual function of male traits}

Male traits could function in intersexual selection, intrasexual selection, or both (Berglund et al., 1996). Females and males exhibited strikingly similar gaze paths when evaluating a displaying male. Similar to the results in this study on peacocks, peahens primarily gazed at the lower display regions of males: at their lower trains, body and legs (Yorzinski et al., 2013). These gaze results suggest that male traits are under selection by both rivals and potential mates. Males did spend more time looking at the upper eyespots and less time looking at the dense feathers in the frontal display compared with females, which could indicate that the upper eyespots play a larger role in rival assessment compared with mate choice and the dense feathers play a larger role in mate choice compared with rival assessment. Both peahens (Yorzinski et al., 2013) and peacocks allocated a similar amount of time toward looking at displaying males versus the environment (males: $27.9 \%$ of time looking at rival male; females: $27.5 \%$ of time looking at potential mate). They both spent over half of their time scanning the environment, which is important given that predation risk can be elevated during rival and mate assessment (Cooper, 1999; Hebets, 2005). We also found a key difference between the gaze patterns of males and females. Peahens spent more time looking at males when they were train rattling (Yorzinski et al., 2013) but males did not (this study), suggesting that train rattling functions in intersexual selection but not intrasexual selection.

Both males and females directed little attention toward the upper train of displaying males. Even though females do not spent much time looking at the upper train, we previously found that they are probably using the upper train to locate potential mates in environments with dense vegetation (Yorzinski et al., 2013). Future experiments will be necessary to assess whether the upper train functions similarly in male-male competition, with males using the upper train to locate rivals in complex environments.

\section{Acknowledgements}

We thank Peter and Martha Klopfer for allowing us to house the birds on their farm. John Pearson provided assistance with the statistical analysis. Bridget Breslin, Samantha Hall, Vassi Hinova, Bailey Goff, Jennifer Lam, Adrian Koehler-Marsh, Megan Orth, Trevor Putt and Sunny Yoon assisted with video analysis.

\section{Competing interests}

The authors declare no competing or financial interests.

\section{Author contributions}

Conceptualization, J.L.Y.; Formal Analysis, J.L.Y. and S.B.; Funding Acquisition, J.L.Y., G.L.P. and M.L.P.; Investigation, J.L.Y.; Visualization, J.L.Y. and S.B.; Writing - Original Draft Preparation, J.L.Y.; Writing - Review \& Editing, J.L.Y., S.B., G.L.P. and M.L.P.

\section{Funding}

This research was funded by the College of Agriculture and Life Sciences at Texas A\&M University, Texas A\&M AgriLife Research, a National Science Foundation graduate research fellowship, an Animal Behavior Society student research grant, the Animal Behavior Graduate Group at UC Davis, the Chapman Memorial Fund, a grant-in-aid of research from the National Academy of Sciences (administered by Sigma-Xi, The Scientific Research Society), a Philanthropic Educational Organization scholar award, and a National Geographic Society/Waitt Foundation grant to J.L.Y. Funding was provided by UC Davis and National Science Foundation grants to G.L.P. (IOS-0925038 and IOS-1258217). The Alfred P. Sloan Foundation and Duke Lemur Center provided funding to M.L.P.

\section{Data availability}

Model files and additional plots are available at https://github.com/bykau/peacock.

Supplementary information

Supplementary information available online at

http://jeb.biologists.org/lookup/doi/10.1242/jeb.150946.supplemental

\section{References}

Andersson, M. (1994). Sexual Selection. Princeton: Princeton University Press Berglund, A., Bisazza, A. and Pilastro, A. (1996). Armaments and ornaments: an evolutionary explanation of traits of dual utility. Biol. J. Linn. Soc. 58, 385-399.

Borgia, G. (1979). Sexual selection and the evolution of mating systems. In Sexual Selection and Reproductive Competition in Insects (ed. M. S. Blum and N. A. Blum), pp. 27-49. New York: Academic Press.

Borgia, G. and Coleman, S. W. (2000). Co-option of male courtship signals from aggressive display in bowerbirds. Proc. R. Soc. B Biol. Sci. 267, 1735-1740.

Cooper, W. E., Jr. (1999). Tradeoffs between courtship, fighting, and antipredatory behavior by a lizard, Eumeces laticeps. Behav. Ecol. Sociobiol. 47, 54-59.

Dakin, R. and Montgomerie, R. (2011). Peahens prefer peacocks displaying more eyespots, but rarely. Anim. Behav. 82, 21-28. 
Dakin, R., McCrossan, O., Hare, J. F., Montgomerie, R. and Amador Kane, S. (2016). Biomechanics of the peacock's display: How feather structure and resonance influence multimodal signaling. PLOS ONE 11, e0152759.

Darwin, C. R. (1871). The Descent of Man and Selection in Relation to Sex. London: Murray.

Dukas, R. (2002). Behavioural and ecological consequences of limited attention. Philos. Trans. R. Soc. B Biol. Sci. 357, 1539-1547.

Dukas, R. and Kamil, A. C. (2000). The cost of limited attention in blue jays. Behav. Ecol. 11, 502-506.

Geist, V. (1971). The Mountain Sheep: A Study in Behaviour and Evolution. Chicago: Chicago University Press.

Griggio, M., Hoi, H. H. and Pilastro, A. (2010). Plumage maintenance affects ultraviolet colour and female preference in the budgerigar. Behav. Process 84, 739-744.

Hebets, E. A. (2005). Attention-altering signal interactions in the multimodal courtship display of the wolf spider Schizocosa uetzi. Behav. Ecol. 16, 75-82.

Loffredo, C. A. and Borgia, G. (1986). Sexual selection, mating systems, and the evolution of avian acoustical display. Am. Nat. 128, 773-794.

Loyau, A., Jalme, M. S. and Sorci, G. (2005). Intra- and intersexual selection for multiple traits in the peacock (Pavo cristatus). Ethology 111, 810-820.

Maurer, D. (1975). Infant visual perception: methods of study. In Infant Perception: From Sensation to Cognition, Vol. 1, Basic Visual Processes (ed. L. B. Cohen and P. Salapatek), pp. 1-76. New York, NY: Academic.

McCullough, E. L. and Simmons, L. W. (2016). Selection on male physical performance during male-male competition and female choice. Behav. Ecol. 27, 1288-1295.

Moore, A. J. (1990). The evolution of sexual dimorphism by sexual selection: The separate effects of intrasexual selection and intersexual selection. Evolution 44 315-331.
O'Brian, W. J. and Showalter, J. J. (1993). Effects of current velocity and suspended debris on the drift feeding of Arctic greylings. Trans. Am. Fish. Soc. 122, 609-615.

Petrie, M., Halliday, T. and Sanders, C. (1991). Peahens prefer peacocks with elaborate trains. Anim. Behav. 41, 323-331.

Rosenthal, G. G. (2007). Spatiotemporal dimensions of visual signals in animal communication. Annu. Rev. Ecol. Evol. Syst. 38, 155-178.

von Schantz, T., Göranson, G., Andersson, G., Fröberg, I., Grahn, M., Helgée, A. and Wittzell, H. (1989). Female choice selects for a viability-based male trait in pheasants. Nature 337, 166-169.

Vreven, D. and Blough, P. M. (1998). Searching for one or many targets: effects of extended experience on the runs advantage. J. Exp. Psychol. Anim. Behav. Process 24, 98-105.

Walther, B. A. (2003). Do peacocks devote maintenance time to their ornamental plumage? Time budgets of male blue peafowl Pavo cristatus. Lundiana 4, 149-154.

Walther, B. A. and Clayton, D. H. (2005). Elaborate ornaments are costly to maintain: evidence for high maintenance handicaps. Behav. Ecol. 16, 89-95.

Yorzinski, J. L. and Platt, M. L. (2014). Selective attention in peacocks during predator detection. Anim. Cogn. 17, 767-777.

Yorzinski, J. L., Patricelli, G. L., Babcock, J. S., Pearson, J. M. and Platt, M. L. (2013). Through their eyes: selective attention in peahens during courtship. J. Exp. Biol. 216, 3035-3046.

Yorzinski, J. L., Patricelli, G. L., Platt, M. L. and Land, M. F. (2015). Eye and head movements shape gaze shifts in Indian peafowl. J. Exp. Biol. 218, 3771-3776.

Zahavi, A. (1991). On the definition of sexual selection, fisher's model, and the evolution of waste and of signals in general. Anim. Behav. 42, 501-503. 